\title{
Cuidado de indivíduos com diabetes mellitus: a consulta de enfermagem na perspectiva de enfermeiras
}

Care of individuals with diabetes mellitus: nursing consultation from the perspective of nurses

Atención de personas con diabetes mellitus: consulta de enfermería desde la perspectiva de las enfermeras

\section{Claudia Moresco Peliser Beal', Valéria Silvana Faganello Madureira ${ }^{\mathrm{II}}$, Jeane Barros de Souza ${ }^{\mathrm{III}}$, Liane Colliselliv ${ }^{\mathrm{v}}$, Larissa Hermes Thomas Tombiniv ${ }^{\mathrm{v}}$, Daniela Savi Geremia ${ }^{\mathrm{vI}}$}

\begin{abstract}
Resumo: Objetivo: conhecer a perspectiva de enfermeiras sobre a consulta de enfermagem no cuidado com indivíduos com Diabetes mellitus. Método: estudo exploratório descritivo qualitativo desenvolvido em município de Santa Catarina, Brasil. Participaram seis enfermeiras de unidades básicas e serviço de referência. Os dados foram coletados com entrevistas semiestruturadas, entre junho e agosto de 2015. A análise seguiu a técnica de Análise Temática. Resultados: quando feita, a consulta de enfermagem é incompleta, focalizada em queixas, repetitiva e vinculada à renovação de receitas, havendo falta de profissionais, alta demanda e preocupação em atender rapidamente durante a assistência. Conclusão: persiste o desafio da realização da consulta de enfermagem. Neste sentido, o dimensionamento adequado de pessoal da equipe é importante, prevendo possíveis faltas ao trabalho. Articulação teoria e prática e reorganização do processo de trabalho para atenção integral a indivíduos com diabetes mellitus são essenciais para que a consulta de enfermagem seja utilizada no cuidado.

Descritores: Cuidados de enfermagem; Enfermagem no consultório; Diabetes mellitus; Centros de saúde; Atenção primária à saúde
\end{abstract}

Abstract: Objective: to know the perspective of nurses on the nursing consultation in the care of individuals with Diabetes mellitus. Method: exploratory qualitative descriptive study developed in the city of Santa Catarina, Brazil.

\footnotetext{
IEnfermeira. Especialização em Urgência e Emergência. Marcelino Ramos, Rio Grande do Sul, Brasil, e-mail: clau.moresco.peliser@gmail.com ORCID: http://orcid.org/0000-0002-1684-9434

II Enfermeira. Doutora em Enfermagem. Universidade Federal da Fronteira Sul (UFFS) Chapecó, Santa Catarina, Brasil, e-mail: valeriamadureira2005@hotmail.com ORCID: http://orcid.org/0000-0001-7990-3613

III Enfermeira. Doutora em Ciências. Universidade Federal da Fronteira Sul (UFFS), Chapecó, Santa Catarina, Brasil, e-mail: jeane.souza@uffs.edu.br ORCID: http://orcid.org/0000-0002-0512-9765

IV Enfermeira. Mestre em Enfermagem. Docente aposentada do curso de Enfermagem da Universidade Federal da Fronteira Sul (UFFS), Chapecó, Santa Catarina, Brasil, e-mail: colliselli.liane@gmail.com ORCID: http://orcid.org/0000-0002-2774-5083

Enfermeira. Mestre em Enfermagem. Universidade Federal da Fronteira Sul (UFFS), Chapecó, Santa Catarina, Brasil, larissa.tombini@uffs.edu.br ORCID: http://orcid.org/0000-0002-6699-495

VI Enfermeira. Doutora em Saúde Coletiva. Universidade Federal da Fronteira Sul (UFFS), Chapecó, Santa Catarina, Brasil, e-mail: daniela.geremia@uffs.edu.br ORCID: http://orcid.org/0000-0003-2259-7429
} 
Six nurses from basic units and reference service participated. Data were collected through semi-structured interviews, between June and August 2015. The analysis followed the Thematic Analysis technique. Results: when done, the nursing consultation is incomplete, focused on complaints, repetitive and linked to the renewal of prescriptions, with a lack of professionals, high demand and concern to attend quickly during assistance. Conclusion: the challenge of carrying out the nursing consultation persists. In this sense, the adequate sizing of team personnel is important, providing for possible absences from work. Articulation of theory and practice and reorganization of the work process for comprehensive care for individuals with diabetes mellitus are essential for the nursing consultation to be used in care.

Descriptors: Nursing care; Office nursing; Diabetes mellitus; Health centers; Primary health care

Resumen: Objetivo: conocer la perspectiva de las enfermeras sobre la consulta de enfermería en el cuidado de personas con Diabetes mellitus. Método: estudio descriptivo cualitativo exploratorio desarrollado en la ciudad de Santa Catarina, Brasil. Participaron seis enfermeras de unidades básicas y servicio de referencia. Los datos fueron recolectados a través de entrevistas semiestructuradas, entre junio y agosto de 2015. El análisis siguió la técnica de Análisis temático. Resultados: cuando se realiza, la consulta de enfermería es incompleta, enfocada en quejas, repetitiva y vinculada a la renovación de recetas, con falta de profesionales, alta demanda y preocupación por atender rápidamente durante la asistencia. Conclusión: el desafío de llevar a cabo la consulta de enfermería persiste. En este sentido, el tamaño adecuado del personal del equipo es importante, ya que prevé posibles ausencias del trabajo. La articulación de la teoría y la práctica y la reorganización del proceso de trabajo para la atención integral de las personas con diabetes mellitus son esenciales para la consulta de enfermería que se utilizará en la atención.

Descriptores: Atención en enfermería; Enfermería de consulta; Diabetes mellitus; Centros de salud; Atención primaria de salud

\section{Introdução}

O Diabetes mellitus (DM) é uma doença crônica considerada pandêmica, com custos humanos, sociais e econômicos ligados à mortalidade precoce e a incapacidades resultantes de complicações. ${ }^{1}$ Trata-se de distúrbio metabólico de múltipla etiologia, caracterizado por hiperglicemia, transtornos do metabolismo de gorduras, proteínas e carboidratos, resultante de deficiência na secreção e ou na ação de insulina, o que demanda acompanhamento, tratamento e cuidados para prevenção de outros agravos decorrentes de níveis persistentemente elevados de glicemia. ${ }^{2}$

No Brasil, considerando a população maior ou igual a 18 anos, a pesquisa de vigilância de fatores de risco e proteção para doenças crônicas por inquérito telefônico ${ }^{3}$ identificou que $7,7 \%$ dos pesquisados referiram diagnóstico médico para diabetes $(8,1 \%$ de mulheres; $7,1 \%$ de homens) e, em ambos os sexos, a frequência da condição crônica aumentou com a idade e diminuiu entre 
3 | Beal CMP, Madureira VSF, Souza JB, Colliselli L, Tombini LHT, Geremia DS

àqueles com maior escolaridade. Em Florianópolis, capital do estado de Santa Catarina, a frequência foi de $6,7 \%$, variando entre $6,1 \%$ no sexo masculino e $7,2 \%$ no feminino. ${ }^{4}$

Como Doença Crônica Não Transmissível (DCNT), o DM interfere na qualidade de vida e na saúde das populações, assim como na organização dos processos de trabalho das equipes nos serviços de atenção à saúde. A carga dessas doenças é crescente no mundo, o que pode ser atribuído ao estilo de vida contemporâneo, a mudanças epidemiológicas e demográficas, bem como à própria globalização. ${ }^{5}$

Ciente disso e em coerência com as iniciativas internacionais, em 2011 foi lançado o Plano de Ações Estratégicas para o Enfrentamento das DCNT no Brasil, com o objetivo de preparar o país para prevenir e controlar tais doenças entre os anos de 2011 e 2022. Para isso propunha o desenvolvimento e implementação de políticas públicas efetivas, visando à redução da morbidade, da mortalidade e de incapacidade delas decorrentes. ${ }^{6}$

A implementação do Plano tem demonstrado avanços nas ações de vigilância, promoção da saúde e cuidado integral, a exemplo da construção da Rede de Atenção às Pessoas com Doenças Crônicas proposta em 2012. Entretanto, apesar do progresso observado, muito ainda há a ser feito, especialmente no que se refere à formação e ao processo de trabalho das equipes para assistência resolutiva. ${ }^{7}$ As metas do Plano estão sendo cumpridas, porém é necessário monitoramento contínuo para ampliação das ações já implementadas e revisão das estratégias utilizadas. ${ }^{8}$

É importante destacar que sedentarismo, obesidade e longevidade podem contribuir para o crescimento das DCNT e demandam estratégias que incluem, além do tratamento medicamentoso, a participação e adesão comportamental regular do indivíduo. Neste contexto, é essencial o envolvimento e estabelecimento de vínculos entre a equipe multiprofissional e o indivíduo que vivencia o DM, com vistas ao efetivo controle da doença. ${ }^{9}$

No contexto do cuidado ao indivíduo com DM, apresenta-se o acompanhamento multiprofissional na atenção primária à saúde (APS), com destaque à Estratégia Saúde da 
Cuidado de indivíduos com diabetes mellitus: a consulta de enfermagem na perspectiva... | 4

Família (ESF), campo fértil para a promoção da saúde e da qualidade de vida de indivíduos e coletivos. Neste sentido, evidencia-se a importância do enfermeiro e demais membros da equipe na realização de ações de educação em saúde, oportunizando que o indivíduo adquira autonomia sobre seus cuidados, tornando-se capaz de compreender, avaliar e tomar decisões sobre a sua doença. ${ }^{9}$

Considerada a perspectiva da atenção integral ao DM, o enfermeiro se coloca como profissional essencial para o cuidado em todos os níveis de atenção e componentes de uma rede integrada. Porém, seu papel se destaca na APS, em razão das possibilidades de prevenção da doença, de detecção precoce e de acompanhamento e avaliação dos já acometidos. O trabalho do enfermeiro neste nível de atenção é estratégico e fundamental, pelo potencial de identificação das necessidades de saúde dos indivíduos e realização de estratificação de riscos, que subsidiarão a organização do cuidado em rede. ${ }^{10}$

Neste sentido, a Política Nacional de Atenção Básica (PNAB) ${ }^{11}$ inclui, entre as atribuições deste profissional, a realização da consulta de enfermagem e procedimentos, a solicitação de exames complementares e a prescrição de medicações conforme protocolos, diretrizes clínicas e terapêuticas. Além disso, merecem destaque a estratificação de risco e a elaboração de plano de cuidados para aqueles que, no território, vivem com condições crônicas de saúde. ${ }^{12}$

No exercício da profissão, o enfermeiro deve operacionalizar seu atendimento pela implementação do processo de enfermagem (PE), realizando-o de forma deliberada e sistemática, em qualquer ambiente onde ocorra o cuidado profissional de enfermagem. Na APS, esse instrumento é usualmente chamado de Consulta de Enfermagem (CE) e tem cinco passos: Histórico de Enfermagem (obtenção de informações do indivíduo e família, bem como exame físico); Diagnóstico de Enfermagem (elaboração dos conceitos diagnósticos sobre a situação de saúde do indivíduo); Planejamento de Enfermagem (intervenções a serem realizadas e resultados esperados); Implementação (realização das intervenções estabelecidas) e Avaliação de 
5 | Beal CMP, Madureira VSF, Souza JB, Colliselli L, Tombini LHT, Geremia DS

Enfermagem (processo que avalia se o resultado esperado foi alcançado e se há necessidade de modificações nas intervenções). ${ }^{12}$

Como elemento importante do ser-saber-fazer da enfermagem, a CE não é tema novo. Há décadas é considerada como aplicação do PE na assistência profissional ao "indivíduo não hospitalizado, aparentemente sadio ou ao doente em tratamento ambulatorial". ${ }^{13: 53}$ Atualmente, o processo de enfermagem (PE), que se expressa na atenção não hospitalar como CE, compõe a sistematização da assistência de enfermagem (SAE), como "eixo fundante e estruturante da construção do conhecimento e da prática profissional (ensino, assistência, pesquisa e gestão/gerenciamento)” da enfermagem. ${ }^{14: 5}$ Como tal, demanda diferentes habilidades, capacidades, estudo, flexibilidade, criatividade e inovação para que o cuidado seja mais próximo da realidade de vida e das necessidades das pessoas. Entretanto, apesar da legislação vigente e de ser uma temática presente nas pesquisas na área da enfermagem, ainda não está consolidada nos cenários em que o cuidado é desenvolvido. ${ }^{14}$

A partir do contexto delineado emergiu a seguinte questão de pesquisa: qual a perspectiva de enfermeiras sobre a CE no cuidado de indivíduos com DM? Neste âmbito, urge a necessidade de ampliar a discussão sobre o cuidado de enfermagem a indivíduos diagnosticados com DM, com vistas a aprimorar a assistência à saúde. Assim, este artigo objetiva conhecer a perspectiva de enfermeiras sobre a CE no cuidado de indivíduos com DM.

\section{Método}

Trata-se de estudo exploratório descritivo com abordagem qualitativa, desenvolvido em três Unidades Básicas de Saúde (UBS) e em um Centro de Referência (CR) da rede de serviços próprios da Secretaria Municipal de Saúde de um município de médio porte do oeste de Santa Catarina (SC), Brasil. Tais locais foram escolhidos por configurarem espaços de atenção e cuidado a indivíduos com DM e por constituírem ambientes de práticas acadêmicas e de 
Cuidado de indivíduos com diabetes mellitus: a consulta de enfermagem na perspectiva... | 6

desenvolvimento de projeto de extensão de um curso de graduação em enfermagem de uma universidade pública catarinense. É importante esclarecer que, os indivíduos com DM vinculados às UBS são encaminhados ao CR para consulta com especialidades médicas onde, da mesma forma, são atendidos por enfermeiros.

Para inclusão dos participantes foram utilizados os seguintes critérios: ser enfermeira(o) atuante em uma das UBS selecionadas e no $\mathrm{CR}$, prestando assistência há pelo menos um ano a indivíduos com DM. Nas UBS e no CR atuam, ao todo, oito enfermeiras e, delas, seis atenderam aos critérios de seleção, sendo cinco que trabalham em UBS e uma no $C R$, as quais participaram do estudo. Todas as enfermeiras aceitaram participar. A coleta de dados se deu com entrevistas semiestruturadas guiadas por roteiro específico com 14 questões que abordavam sobre o tempo de formação e atuação na Enfermagem, bem como sobre a assistência a indivíduos com DM. As entrevistas foram desenvolvidas no local de trabalho das participantes, após agendamento prévio, no período de 18 de junho a 17 de julho de 2015. O convite para participar e o agendamento das entrevistas foi feito por contato telefônico, no qual o estudo foi apresentado.

Anteriormente à coleta com as participantes, duas entrevistas com outras enfermeiras foram feitas para verificação da adequação do roteiro de questões e para preparo da entrevistadora. Após cada uma das entrevistas, o áudio foi ouvido para que a conduta da entrevistadora fosse avaliada e aprimorada. Após isso, teve início a coleta de dados.

No encontro para as entrevistas com os participantes ocorridos nas UBS e no CR em sala que oferecia privacidade, o estudo foi novamente apresentado e, após esclarecimento de dúvidas, foi assinado o Termo de Consentimento Livre e Esclarecido. Os relatos foram gravados em smartphone, armazenados em computador e integralmente transcritos. Cada encontro teve duração média de 40 minutos. As entrevistas foram conduzidas pela primeira autora deste artigo sob acompanhamento, supervisão e orientação da segunda autora. A coleta de dados foi interrompida quando todas as seis enfermeiras haviam sido entrevistadas. O texto resultante de 
7 | Beal CMP, Madureira VSF, Souza JB, Colliselli L, Tombini LHT, Geremia DS

cada transcrição foi validado com as entrevistadas, as quais o leram e concordaram com o seu teor. Ademais, os Critérios Consolidados para Relatos de Pesquisa Qualitativa (Consolidated Criteria for Reporting Qualitative Research - COREQ) nortearam a elaboração deste artigo como forma de assegurar a qualidade do relato. ${ }^{15}$

Para análise dos dados seguiu-se as diretrizes da análise temática com as seguintes etapas operacionais: ${ }^{16}$ a) pré-análise, em que foi desenvolvido leitura flutuante repetida, no sentido de reconhecer as ideias presentes nas transcrições e compor o corpus da pesquisa; b) exploração do material, com leitura atenta para classificação e agregação dos dados e definição de categorias;

c) Tratamento dos resultados obtidos e interpretação com estabelecimento de relações com a literatura. Desse processo de análise, a convergência de ideias resultou na identificação da temática 'cuidado de enfermagem', com duas categorias e seis subcategorias, as quais seguem representadas e discutidas na próxima seção.

Este estudo seguiu as recomendações da Resolução 466/2012 do Conselho Nacional de Saúde e a coleta dos dados iniciou após a aprovação do protocolo de pesquisa (CAAE nº 45125615.4.0000.5564) pelo Comitê de Ética em Pesquisa, com parecer número 1101819 de 10 de junho de 2015. Na apresentação dos resultados, o anonimato dos participantes foi preservado pela utilização da letra E (entrevistado) seguida de número arábico indicativo da ordem de realização das entrevistas (Ex: E1).

\section{Resultados e discussão}

Nesta seção segue a representação gráfica das categorias e subcategorias identificadas na temática 'Cuidado de Enfermagem’ (Figura 1). 
Figura 1 - Representação gráfica da temática 'cuidado de enfermagem', suas categorias e subcategorias. Santa Catarina, 2015.

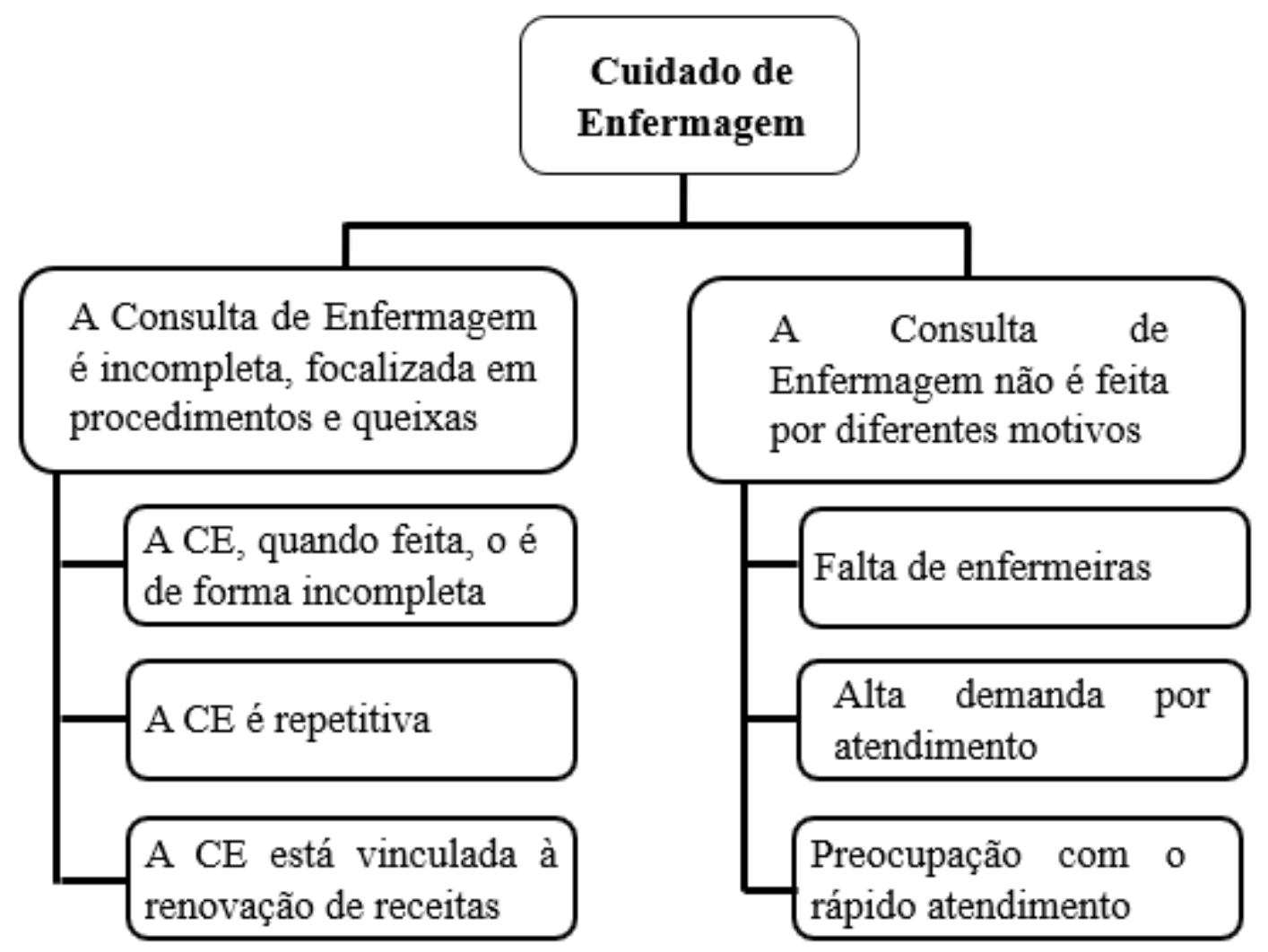

Fonte: elaborado pelos autores

Como demonstrado na Figura 1, a CE não é adotada como prática cotidiana das enfermeiras na atenção a indivíduos com DM, circunstância por elas atribuída a diferentes fatores, tais como a necessidade de suprir a falta de um colega e o desejo de atender rapidamente a todos. A CE é uma tecnologia de assistência central no desempenho do enfermeiro na APS e a expressão 'consulta de enfermagem' é sinônimo de processo de enfermagem quando empregada na atenção primária, ambulatórios, domicílios, escolas e outros serviços que não sejam hospitalares. ${ }^{12,17}$

Como tal, a CE dispõe de legislação específica que a regulamenta. Assim, nos termos da Resolução do Conselho Federal de Enfermagem - COFEN 358/2009, a CE é “[...] instrumento metodológico que orienta o cuidado profissional de enfermagem e a documentação da prática profissional”12:1 e indispensável na SAE em qualquer instituição de saúde. 
9 | Beal CMP, Madureira VSF, Souza JB, Colliselli L, Tombini LHT, Geremia DS

A atenção integral à saúde do indivíduo com condição crônica na perspectiva da rede de atenção exige articulação e comunicação entre os diferentes componentes/serviços e, sobretudo, a atuação profissional de distintos núcleos de saberes e práticas, ${ }^{5}$ com papel central da APS e do enfermeiro neste processo. Na APS, a CE é importante para identificação das necessidades de cuidado de portadores de DCNT, tal como o DM. Nela, dados são coletados, diagnósticos de enfermagem são identificados e condutas são delineadas, de forma que o cuidado seja resolutivo.

\section{Consulta de Enfermagem incompleta, focalizada em procedimentos e queixas}

As participantes revelaram que a "CE, quando feita, o é de forma incompleta", o que está relacionado a diferentes fatores, tais como a presença ou não de complicações:

Tem paciente que tem queixa de ferida no pé. Aí, [a enfermeira] faz exame físico e faz orientação sobre lesão no pé. Não tem queixa, acaba passando batido e ele não sabe muitas vezes os cuidados que deve fazer. (E3)

Se ele tem problema nos pés, alguma dificuldade, as unhas, a gente sempre pergunta; se tem alguma queixa a gente olha, se não tem queixas, não. (E4)

Quando se percebe que o paciente está meio pálido, a gente vai no prontuário eletrônico, investiga, olha os últimos exames. (E6)

Conforme evidenciam os depoimentos, as enfermeiras vinculam sua conduta na atenção a queixas ou a sinais apresentados pelo usuário, o que parece reforçar a ideia de que a assistência prioriza aspectos curativos e sugere forte influência do modelo biomédico na atuação destas profissionais. Ao mesmo tempo evidenciam que o potencial da CE para promover a saúde e prevenir complicações do DM não é completamente utilizado.

A CE envolve diferentes etapas já aqui descritas, o que a coloca como elemento importante da SAE. ${ }^{12}$ É tecnologia essencial no cuidado de enfermagem, que possibilita o direcionamento da assistência, a tomada de decisões no processo de cuidar e a avaliação das 
Cuidado de indivíduos com diabetes mellitus: a consulta de enfermagem na perspectiva... | 10

intervenções. ${ }^{17}$ Ademais, a CE é função privativa do enfermeiro que aumenta sua autonomia no processo de cuidar, possibilitando-lhe avaliar diferentes situações de saúde e doença considerando as condições concretas de vida de cada indivíduo, família e ou comunidade. ${ }^{12}$ Como tal, demanda conhecimento, habilidades e competências específicas.

Assim, pode-se dizer que a realização da CE a indivíduos com DM possibilita um cuidado de enfermagem integral e resolutivo, favorecendo a promoção da saúde e a prevenção do desenvolvimento de complicações, deixando de ter apenas um papel curativo. O cuidado passa a ser baseado nas necessidades identificadas em criteriosa avaliação no histórico de enfermagem, valorizando o conhecimento das condições de vida daquele indivíduo, bem como suas queixas relatadas na consulta, as quais já podem sinalizar complicações do DM. Entretanto, o teor dos depoimentos apresentados sugere que a CE não vem sendo instrumento básico de trabalho na atenção a indivíduos com DM, mesmo sendo considerada obrigatoriedade do enfermeiro desde o ano de $2002 .^{12}$

Estudo desenvolvido no Rio Grande do Sul, com vistas a conhecer como os profissionais da ESF conduziam o processo de cuidar de indivíduos com hipertensão arterial sistêmica e DM, concluiu que a CE não está incluída na assistência rotineira e não houve identificação de registros no prontuário dos usuários. ${ }^{18}$ Já prática assistencial desenvolvida em Criciúma/SC com pessoas com DCNT revelou que a CE fortaleceu o vínculo do enfermeiro com elas, fazendo-as se sentirem acolhidas, mais confiantes e despertando-lhes o desejo de participar de grupo terapêutico, bem como ampliando a possibilidade de aderirem ao tratamento. ${ }^{19} \mathrm{Na}$ mesma direção, pesquisa com 20 enfermeiros da APS de Florianópolis, SC, apontou que a CE é “considerada um instrumento de fortalecimento de vínculo entre o profissional e o usuário, bem como um espaço para o desenvolvimento da autonomia e independência profissional”, embora muitos enfermeiros da APS não a desenvolvam..$^{20: 256}$ 
11 | Beal CMP, Madureira VSF, Souza JB, Colliselli L, Tombini LHT, Geremia DS

Outra pesquisa desenvolvida na mesma cidade identificou que a presença de complicações crônicas, muitas vezes irreversíveis, representou um marco no acompanhamento do indivíduo com DM. ${ }^{21}$ Tais resultados vêm ao encontro do que se obteve no presente estudo e demonstrado nos depoimentos. Ressalta-se que, no DM, as condições de vida e a forma de viver são centrais no cuidado. Por isso, é necessário compreendê-las, o que é possibilitado pelo atendimento individualizado, ${ }^{21}$ proporcionado pela CE.

Outro aspecto a considerar nesta categoria é "na CE, as orientações são sempre as mesmas”, conforme evidenciaram os relatos das enfermeiras:

Na segunda consulta, ele já vem para a enfermeira. Nessa consulta, toda vez a gente aborda os cuidados com a alimentação, medicação, exercício físico. Depois, a gente vai repetindo, reforçando. Toda vez [que o usuário vem] a gente faz hemoglicoteste, uma vez por ano ou mais solicitamos exames para ver como ele está. (E4)

[...] na consulta de rotina, a gente sempre enfoca mais a alimentação, a medicação, incentiva as mulheres a fazerem o preventivo, essas coisas também a gente dá um toque. A consulta seria mais ou menos assim, enfocando alimentação, medicação e exercício físico [....]. A gente repete muitas vezes. (E5)

Os relatos revelam repetição das mesmas orientações a cada novo encontro com o usuário, o que pode estar relacionado ao desenvolvimento incompleto ou ao não desenvolvimento da CE. A falta da anamnese e do exame físico (histórico de enfermagem) faz com que as necessidades, dificuldades e dúvidas dos indivíduos com DM não sejam evidenciadas. Sem obter essas informações e sem avaliar a situação, a enfermeira repassa as mesmas informações de rotina a todos, sem que particularidades e singularidades sejam valorizadas. Assim, adota um comportamento prescritivo e unidirecional de transmissão de conhecimento, o que não contribui para a mudança desejada na situação de saúde e para a resolutividade do cuidado. 
Cuidado de indivíduos com diabetes mellitus: a consulta de enfermagem na perspectiva... | 12

Essa forma de agir reforça a percepção de uma prática fundamentada em um modelo biomédico, com educação em saúde pouco instrumentalizada e baseada na transmissão de conhecimentos e orientações. ${ }^{9}$ Não se trata de desvalorizar as orientações feitas, mas de argumentar a necessidade de que sejam adequadas a cada um de acordo com sua realidade de vida e saúde, como forma de ampliar as possibilidades de integração daquilo que é orientado nos cuidados de saúde cotidianos do indivíduo.

Considerando-se que o enfermeiro é um educador em saúde, espera-se que perceba as necessidades do usuário para que possa desempenhar um papel mediador nesse processo de educação. $^{9}$ Nesse sentido, a CE possibilita que os saberes e a cultura dos envolvidos sejam valorizados, de tal forma que as trocas e os compartilhamentos estabelecidos nesse espaço revertam em promoção da saúde.

A resolução COFEN 358/200912 descreve os objetivos da anamnese ou histórico de enfermagem detalhando inclusive as técnicas a serem utilizadas no exame físico para que o levantamento de informações sobre a saúde do indivíduo seja criterioso. Ou seja, os ordenamentos legais da profissão normatizam o processo de enfermagem como instrumento básico, reflexo do saber científico que fundamenta a disciplina. A CE, realizada nas suas especificidades, viabiliza qualidade do cuidado, pois proporciona uma abordagem singular, apropriada às peculiaridades de cada indivíduo, trazendo benefícios à equipe e ao usuário. ${ }^{10}$

Nos depoimentos, a prática de fazer a “CE na renovação da receita médica” se repete. Uma nova receita é entregue quando o indivíduo busca a unidade de saúde com este fim ou em um encontro de grupo:

A gente aproveita para fazer a consulta quando eles retornam para renovar a prescrição da medicação. Mas não é aquela consulta com todos os passos! [...] olha os exames, se estão em dia ou não, orienta sobre alimentação e renova a receita. (E2) 
A gente faz as consultas de enfermagem na hora que o paciente procura a unidade para renovar a receita. Aí, na triagem, a técnica vê os sinais vitais e, se o índice glicêmico está alterado, a gente pede um controle e exames de rotina anual também. Já olho no prontuário e vejo se está atrasado ou não. Mas se não é primeira consulta, o diabético não quer orientação; quer só a receita porque tem pressa. Claro que a gente não vai só entregar a receita. Sempre conversa. (E3)

Olha, na renovação de receita a gente faz muita coisa! Fazemos o grupo a cada quatro meses e a gente entrega a receita lá. Aí, vê sinais vitais, peso, HGT e já orienta o que fazer se tem alterações. Sempre tem uma palestra com assuntos que a gente considera importantes para eles. Alguns chegam lá e dizem "você pode dar minha receita, que daí eu vou embora?”. Não querem participar. (E4)

Geralmente eles vêm para trocar receita, então a gente já orienta a medicação e pergunta como está usando. (E5)

A CE está intimamente vinculada ao procedimento de renovação/repetição de receitas médicas, as quais são válidas por tempo limitado, o que leva os indivíduos à UBS para renová-las. Entretanto, a atenção da enfermeira, e muitas vezes do usuário, está voltada para a receita. Como resultado, a CE se resume a uma conversa rápida focalizada na medicação, alimentação e, por vezes, na solicitação de exames de rotina. O depoimento de E4 sugere que, no encontro de grupo, o foco é a entrega de uma nova receita, apesar da palestra e da coleta de alguns dados, como sinais vitais e HGT. Além disso, o grupo parece ser uma forma de atender mais pessoas ao mesmo tempo.

Nesta mesma direção, estudo identificou que os cuidados com o diabetes realizados em grupo eram feitos como forma de atender maior número de pessoas, otimizando a agenda profissional. Entretanto, os resultados indicam que a principal atividade desenvolvida foi a entrega de prescrições e, às vezes, de solicitação de exames laboratoriais sem, na maioria das vezes, oportunidade para discutir outras temáticas. Tal situação sugere não valorização das necessidades dos indivíduos com DM, revelando falta de acolhimento e de vínculo, bem como 
Cuidado de indivíduos com diabetes mellitus: a consulta de enfermagem na perspectiva... $\mid 14$

ausência de monitoramento sistemático daqueles que têm a doença com vistas à prevenção e redução de complicações. ${ }^{21}$

Os depoimentos aqui apresentados revelam que o agendamento de CE, pelo menos no caso de DM, não é prática adotada pelas enfermeiras. Ao vincular a CE a outros procedimentos ou atividades, um importante instrumento de monitoramento de indivíduos com DM deixa de ser utilizado, que contribuiria para a promoção da saúde global e para a prevenção de outros agravos relacionados às complicações crônicas. Nesse sentido, o não agendamento da CE interfere na qualidade da atenção e na integralidade do cuidado, pois os atendimentos são realizados para a livre demanda ou aproveitando ocasiões em que o indivíduo busca a instituição de saúde por outro motivo. ${ }^{10}$

Há, entretanto, que se reconhecer o valor das atividades grupais para o compartilhamento de saberes e trocas de experiências tão importantes na educação em saúde, o que demanda planejamento articulado da equipe multidisciplinar. A esse respeito, pesquisa desenvolvida na Região Sul do Brasil $^{20}$ reafirmou a CE como ferramenta importante para educação em saúde em razão de fortalecer o vínculo entre profissional e usuário, além de possibilitar autonomia e independência ao enfermeiro. Na mesma direção, estudo que comparou a efetividade da consulta de enfermagem e das ações educativas em grupo na atenção a indivíduos com DM tipo 2, demonstrou que o atendimento individualizado proporcionado pela CE é recomendado para esclarecer dúvidas e ampliar conhecimento sobre a doença, diminuindo seu impacto na qualidade de vida. A realização de atividades de educação em saúde grupais em conjunto com a CE favorece maior adesão ao autocuidado. ${ }^{22}$ Nesse sentido, trata-se de desenvolver ambas as atividades e não optar por uma delas.

É importante destacar, nos resultados do presente estudo, a utilização do encontro com o indivíduo com DM como uma oportunidade de oferecer informações que despertem temor, na tentativa de motivá-lo a cuidar de sua saúde e seguir as orientações: 
Às vezes eles vão se cuidar quando já tem alguma complicação de rins, de olhos. Eu tento sempre assustar. (E4)

[...] a gente fala da visão, do pé, da cicatrização. Daí a gente mostra um pé diabético, feridas. Isso para conscientizar e assustar um pouquinho em relação às complicações. Se não, eles acabam não se cuidando. (E3)

Agindo assim, a enfermeira se coloca na posição de reguladora de comportamentos, mais do que como profissional chave para a educação em saúde necessária à tomada de decisões autônomas e conscientes por parte dos indivíduos com DM. Sugere uma atuação prescritiva, autoritária e coercitiva, baseada no 'eu disse para fazer. Caso você não faça, terá complicações e a culpa será sua'. Talvez, em razão da falta de tempo e do caráter repetitivo impresso às orientações de enfermagem também ressaltadas pelas participantes, o diálogo entre profissional e indivíduo com DM não seja valorizado como instrumento de trocas de saberes e de construção compartilhada do cuidado. Diálogo pressupõe "relação horizontal de respeito, de troca, de colaboração e de abertura para a escuta”, o que é necessário para estabelecimento de confiança mútua e de vínculo, ambos necessários à efetiva comunicação. 23:18

Como trabalho clínico, a CE é importante para a promoção da saúde, especialmente ao fundamentar-se na escuta atenta e no diálogo para valorizar outros aspectos do viver além dos biológicos. A este respeito, ressalta-se que as decisões sobre o tratamento não podem ser exclusivamente técnicas, pois o outro, no caso o indivíduo com DM, traz consigo seus saberes, sua visão sobre a condição e as práticas de cuidado que já adota, todas referenciadas em suas condições concretas de vida. ${ }^{24}$ Daí a importância de aproximar, em diálogo, os saberes e as práticas do indivíduo, nesse caso com diabetes, com os do profissional, nesse caso o enfermeiro. ${ }^{25}$ Assim, a construção de um conhecimento novo e mais próximo da vida das pessoas é mediado pelo diálogo, essencial à comunicação e que demanda, dentre outros elementos, confiança, respeito e simpatia em uma relação interpessoal horizontal. 
Os saberes e os conteúdos culturais que o indivíduo com DM traz consigo se refletem na forma de viver e nos cuidados de saúde, interditando algumas práticas e estimulando outras, por exemplo, no que se refere à alimentação. A CE possibilita conhecê-los e valorizá-los para que, no encontro com o saber profissional, o cuidado seja conjuntamente delineado e possa ser incorporado no cotidiano. Escutar atentamente o outro é essencial, o que é oportunizado pela CE, na qual o enfermeiro se volta ao outro, dedicando-lhe tempo maior do que a atenção direcionada às possíveis queixas que tenha.

\section{A Consulta de Enfermagem não é feita por diferentes motivos}

A "falta de profissionais enfermeiros" foi indicada pelas pesquisadas como um dos motivos para a não realização da CE. Essa situação é considerada como responsável pela diminuição da qualidade do atendimento evidenciada pela impossibilidade de desenvolver a CE com todas as fases:

A gente convive com situações onde um colega está de atestado, outro está de férias. Já aconteceu comigo. Então, você faz assistência de três áreas de ESF ao invés de uma. E aí, o que que acontece? A qualidade do atendimento cai, porque você não faz uma consulta de enfermagem ideal, com aquele exame físico ideal que poderia fazer se tivesse disponibilidade de tempo. (E1)

[...] é difícil fazer la CE] como a gente gostaria. Primeiro sempre está faltando profissionais enfermeiros. A gente fica em duas com três equipes e precisa dar conta de atender [...]. (E2)

Às vezes é uma licença maternidade e, aí, falta enfermeiro. Eu mesma já assumi a coordenação porque faltava enfermeiro para isso. Já cobri na parte da assistência também. (E5)

A falta de profissionais não significa que não haja outros enfermeiros vinculados ao serviço. As ausências decorrem de atestados, férias ou licenças, situações que sobrecarregam o 
profissional que continua atuando, pois não há disponibilização de outro para suprir a necessidade no período de afastamento. O absenteísmo de membros da equipe sobrecarrega o enfermeiro, pois muitas vezes impõe que abandone suas atividades para assumir as que cabem àquele que está ausente. ${ }^{26}$ Há também a identificação que a "alta demanda por atendimento prejudica a realização da CE”:

A gente tem muita demanda para passar todas as informações em consulta de enfermagem. Depende o dia, a gente consegue, mas tem dias que não, por causa da demanda. (E3)

Tem dia que não tem muita gente e eu atendo 30 e poucas pessoas. Então, como é que você vai fazer tudo certinho? A gente vai pelo prático. Quando é a primeira consulta, que a pessoa iniciou o tratamento para o diabetes, você dedica mais tempo. Depois você só reforça. (E4)

Quando está mais calmo, eu até consigo fazer. Porque aqui é livre demanda. Tem dias que tem muita gente, aí é claro que não dá. A gente abrevia. (E6)

Entretanto, aliada à alta demanda e aos atropelos cotidianos está a "preocupação em atender rapidamente a todos”, para que o indivíduo não permaneça muito tempo aguardando. Nesse contexto, a CE, especialmente àqueles usuários já conhecidos, é vista como desperdício de tempo:

[...] na primeira hora da manhã já tinha 10 pacientes. Então, se você contabilizar o tempo para atender 10 pacientes significa que aqueles que chegaram às 7:30, alguns saíram quase às 10 horas da manhã. Então, se veio um diabético lá no terceiro [usuário a ser atendido], que você tinha mais sete pra atender depois, você não vai perder meia hora, uma hora fazendo uma consulta de enfermagem para um paciente que você já conhece corriqueiramente. Se é um paciente novo, a conduta muda muito. (E3)

O depoimento revela um pouco da influência que a organização do processo de trabalho da enfermeira na UBS exerce sobre a realização da CE. A falta de profissionais, a alta demanda 
Cuidado de indivíduos com diabetes mellitus: a consulta de enfermagem na perspectiva... $\mid 18$

de usuários e a preocupação em atender rapidamente formam, portanto, uma tríade de motivos que levam à não realização da CE, inviabilizando um atendimento de maior qualidade. Como efeito cascata, observa-se que a falta de profissionais direciona a alta demanda ao profissional atuante, que atende os usuários de forma rápida para que os demais não aguardem por muito tempo. Nesse cenário, a CE é considerada como consumidora ou como perda de tempo.

Estudo desenvolvido no Sul do Brasil observou que o absenteísmo de membros da equipe de enfermagem gera sobrecarga ao enfermeiro, que geralmente assume para si o cumprimento das atividades, prejudicando sua atuação e levando-o a abandonar aquelas atividades que lhe são privativas. Somam-se a isso as funções administrativas que desenvolve e a falta de espaço físico como empecilho ao desenvolvimento das atividades assistenciais que lhe cabem, tal como a CE. ${ }^{20}$

A alta procura e a falta de tempo disponível para o atendimento são importantes fatores que influenciam a realização ou não da CE, prática que exige dedicação de tempo variável de acordo com as necessidades de uma primeira consulta, de acompanhamento e de acolhimento à demanda espontânea. ${ }^{17} \mathrm{O}$ papel do enfermeiro no acompanhamento de indivíduos com DM inclui rastreamento, prevenção de complicações, intensificação do controle glicêmico e atividades de educação, o que exige habilidades e competências técnico-científicas para desempenhá-lo adequadamente. Neste sentido, desponta a relevância da formação deste profissional para que tenha tais habilidades e competências que possibilitem sua atuação como generalista no gerenciamento da atenção com condições crônicas de saúde, atentando para a integralidade, a equidade e a universalidade do cuidado. ${ }^{9}$

O enfermeiro desenvolve atividades burocráticas por vezes excessivas, o que resulta em sobrecarga de trabalho criando dificuldades para seu envolvimento na assistência. Porém, esse profissional desenvolve atenção de saúde de qualidade quando assume funções clínicas e, além disso, tem preparo para "funções de coordenação com gestão clínica e de casos, gerenciando o cuidado na rede". ${ }^{26: 5}$ 
Os depoimentos revelam também que a não realização da CE como método científico para o cuidado em enfermagem está relacionada à tríade de motivos já apresentada. As participantes mencionam uma consulta ideal, aprendida durante sua formação, mas que não conseguem colocar em prática no seu cotidiano. Impossibilitadas de aplicar a CE da forma como aprenderam, não a utilizam rotineiramente no cuidado.

[...] aquela consulta de enfermagem ideal, que a gente aprende na faculdade nem sempre é possível colocar no dia-a-dia, na prática assistencial. (E1)

Eu não sigo aquele padrão de consulta há muito tempo, porque eu acho que no posto de saúde, em qualquer lugar, você não consegue fazer aquilo! Até porque você tem mais experiência e, só de olhar para o paciente, você já sabe como ele se comporta, o que ele faz. Eu estou aqui há quase 8 anos, então eu conheço quase todos os pacientes. (E4)

A gente avalia a pele, os locais de aplicação da insulina, os pés. Não é mais aquele exame físico acadêmico, abre a boca, olha a mucosa, mas a gente avalia [...]. (E6)

O abandono da CE aprendida no processo de formação é também atribuído à experiência obtida pela enfermeira no cuidado aos indivíduos com DM e com outros usuários, tal como revela a participante E4. Cabe ressaltar que a experiência adquirida no trabalho contribui para agilizar a assistência e a avaliação de saúde. Porém, é preciso ter cuidado para que a proximidade entre profissionais e usuários, possibilitada pela ESF, não leve a conclusões e a ações que desconsideram a situação do indivíduo, sem base em avaliação criteriosa da sua condição de saúde e de vida.

A identificação de situações baseadas apenas no 'olhar' para o indivíduo 'já conhecido' sugere uma maneira de atuar guiada mais pelo senso comum do que pela ciência, o que pode conduzir a interpretações equivocadas e a negligência na atenção. A CE aprendida na graduação segue as finalidades da formação ampla e generalista, sendo executada em condições ideais e 
Cuidado de indivíduos com diabetes mellitus: a consulta de enfermagem na perspectiva... | 20

com tempo necessário para a aprendizagem. A inserção do seu exercício no cotidiano do trabalho em enfermagem na APS, ou em qualquer outro espaço, demanda adaptações, mas não dispensa a coleta de informações subjetivas e objetivas do indivíduo para orientar o cuidado integral e resolutivo.

Entretanto, os depoimentos apontam para um distanciamento entre o ensino da enfermagem e a realidade dos serviços de saúde. É importante a correlação entre teoria e prática e, neste sentido, uma formação sólida é condição necessária para que os enfermeiros tenham embasamento e conhecimento científico para enfrentar as mais diversas situações nas rotinas de trabalho. Ao adotar a CE como tecnologia de cuidado para a prática profissional da enfermagem, ${ }^{17}$ o enfermeiro diferencia sua atuação e pode, no atendimento individual e personalizado, direcionar suas condutas às peculiaridades e necessidades cada indivíduo aumentando a qualidade do cuidado, bem identificar aspectos importantes para abordagem em grupo.

Ao final, ressalta-se como limite deste estudo o fato de não ter incluído enfermeiras(os) atuantes na atenção a indivíduos com DM de todas as Unidades Básicas de Saúde do município. Tal fato oferece apenas um recorte da realidade da atenção, que poderia ter alcançado resultados diferentes caso um enfermeiro de cada UBS tivesse sido entrevistado

\section{Considerações finais}

Os resultados obtidos demonstram que a realização e operacionalização da CE representa um desafio que encontra obstáculos para ser superado. Um deles é a sobrecarga de trabalho do enfermeiro, o qual acumula atividades assistenciais e de gestão com a necessidade de assumir atividades de outro colega em razão de atestados, férias, licenças e outros. Nesse sentido, acredita-se que o dimensionamento pessoal da equipe mereça especial atenção dos gestores. 
A CE não é incluída como atividade essencial no acompanhamento do indivíduo com DM. Nesse cenário, deixa de cumprir seu papel de orientadora do cuidado e perde importância como instrumento para uma atenção voltada à melhoria da condição de saúde do indivíduo, seja para a prevenção, detecção precoce e ou adiamento de complicações. As enfermeiras não se apropriaram dessa tecnologia que lhes abre possibilidades de delinear os caminhos do cuidado e inovar, mesmo dentre tantos protocolos e normativas já instituídas na atenção a indivíduos com DM. A CE ainda não é implementada como espaço de encontro, de vínculos, de trocas, de educação em saúde e de empoderamento.

Como elemento da SAE na APS, a adoção da CE como tecnologia de cuidado possibilita a qualificação da assistência a indivíduos com DM, ampliando a autonomia e a visibilidade da atuação do enfermeiro, as quais há muito tempo estão na pauta das lutas da profissão. A organização da demanda para atendimento de indivíduos com DM poderia tornar o cuidado mais efetivo, valorizando o papel do enfermeiro na equipe multiprofissional. Porém, há a possibilidade de novos estudos nessa área, que ainda tem muito a explorar para a viabilização de aperfeiçoamento da equipe e da qualidade da assistência, visto que o papel do enfermeiro ainda precisa ser mais evidenciado e discutido, a fim de haver maior valorização e incentivo à categoria.

\section{Referências}

1. International Diabetes Federation (IDF). Atlas. $8^{\mathrm{th}}$ ed. Brussels: International Diabetes Federation; 2017.

2. Sociedade Brasileira de Diabetes. Diretrizes da Sociedade Brasileira de Diabetes 2017-2018. São Paulo: Clannad; 2017.

3. Ministério da Saúde (BR), Secretaria de Vigilância em Saúde, Departamento de Análise em Saúde e Vigilância de Doenças não Transmissíveis. Vigitel Brasil 2018: vigilância de fatores de risco e proteção para doenças crônicas por inquérito telefônico: estimativas sobre frequência e distribuição sociodemográfica de fatores de risco e proteção para doenças crônicas nas capitais dos 26 estados brasileiros e no Distrito Federal em 2018. Brasília (DF): Ministério da Saúde; 2019.

4. Ministério da Saúde (BR), Secretaria de Vigilância em Saúde, Departamento de Vigilância de Doenças 
Cuidado de indivíduos com diabetes mellitus: a consulta de enfermagem na perspectiva... | 22

e Agravos não Transmissíveis e Promoção da Saúde. Vigitel Brasil 2016: vigilância de fatores de risco e proteção para doenças crônicas por inquérito telefônico: estimativas sobre frequência e distribuição sociodemográfica de fatores de risco e proteção para doenças crônicas nas capitais dos 26 estados brasileiros e no Distrito Federal em 2016. Brasília (DF): Ministério da Saúde; 2017.

5. Malta DC, Silva AG, Teixeira RA, Machado IE, Coelho MRS, Hartz ZMA. Avaliação do alcance das metas do plano de enfrentamento das doenças crônicas não transmissíveis no Brasil, 2011-2022. Anais IHMT. 2019;18(37):S9-16. doi: 10.25761/anaisihmt.316

6. Ministério da Saúde (BR), Departamento de Análise de Situação de Saúde. Plano de ações estratégicas para o enfrentamento das doenças crônicas não transmissíveis (DCNT) no Brasil 2011-2022 [Internet]. Brasília (DF): Ministério da Saúde; 2011 [acesso em 2019 jul 10]. Disponível em: http://bvsms.saude.gov.br/bvs/publicacoes/plano_acoes_enfrent_dcnt_2011.pdf

7. Malta DC, Oliveira TP, Santos MAS, Andrade SSCA, Silva MMA. Avanços do plano de ações estratégicas para o enfrentamento das doenças crônicas não transmissíveis no Brasil, 2011-2015. Epidemiol Serv Saúde. 2016;25(2):373-90. doi: 10.5123/s1679-49742016000200016

8. Costa FBC, Branco JGGO, Aguiar FAR, Silva Júnior GB, Saintrain MVL, Catrib AMF. Avanços para redução da morbimortalidade das doenças crônicas não transmissíveis na população brasileira. Rev Gerenc Políticas Salud. 2019;18:1-19. doi: 10.11144/Javeriana.rgps18-37.armd

9. Coelho MCVS, Almeida CAPL, Silva ARV, Moura LKB, Feitosa LGGC, Nunes LB. Training in diabetes education: meanings attributed by primary care nurses. Rev Bras Enferm. 2018;71(Suppl 4):16118. doi: 10.1590/0034-7167-2017-0792

10. Oliveira SKPO, Queiroz APO, Matos DPM, Moura AF, Lima FET. Temas abordados na consulta de enfermagem: revisão integrativa da literatura. Rev Bras Enferm [Internet]. 2012 [cited 2019 Sept 10];65(1):155-61. Available from: http://www.scielo.br/pdf/reben/v65n1/23.pdf

11. BRASIL. Ministério da Saúde. Portaria no 2.436, de 21 de setembro de 2017. Aprova a Política Nacional de Atenção Básica, estabelecendo a revisão de diretrizes para a organização da Atenção Básica, no âmbito do Sistema Único de Saúde (SUS). Diário Oficial da União, Brasília, 22 set. 2017. Seção 1, p. 68-76.

12. Conselho Federal de Enfermagem (COFEN). Resolução COFEN 358/2009 [Internet]. 2009 [acesso em 2019 jul 15]. Disponível em: http://www.cofen.gov.br/resoluo-cofen-3582009_4384.html

13. Horta WA. Consulta de enfermagem. Rev Esc Enferm USP [Internet]. 1975 [acesso em 2020 jun 08];9(3):53-7. Disponível em: https://www.scielo.br/pdf/reeusp/v9n3/0080-6234-reeusp-9-3-053.pdf

14. Garcia TR. Sistematização da assistência de enfermagem: aspecto substantivo da prática profissional. Esc Anna Nery. 2016;20(1):5-6. doi: 10.5935/1414-8145.20160001

15. Patias ND, Von Hohendorff J. Critérios de qualidade para artigos de pesquisa qualitativa. Psicol 
Estud. 2019;24(e43536):1-14. doi: 10.4025/psicolestud.v24i0.43536

16. Minayo MC. O desafio do conhecimento: pesquisa qualitativa em saúde. 14ª ed. São Paulo: Hucitec; 2014.

17. Dantas CN, Santos VEP, Tourinho FSV. A consulta de enfermagem como tecnologia do cuidado à luz dos pensamentos de Bacon e Galimberti. Texto Contexto Enferm. 2016;25(1):e2800014. doi: 10.1590/01040707201500002800014

18. Curioletti RM, Colliselli L, Madureira VSF, Tombini LHT. O cuidado a hipertensos e diabéticos na perspectiva da Política Nacional de Humanização. J Nurs Health. 2018;8(1):e188110. doi: 10.15210/jonah.v8i1.12495

19. Mafra LR, Birolo IVB, Tomasi CD, Ceretta LB, Soratto J, Losso ARS. Prática assistencial de enfermagem para portadores de doenças crônicas não transmissíveis e uma Estratégia de Saúde da Família de Criciúma - SC: um relato de experiência. Inova Saúde [Internet]. 2018 [acesso 2019 set 10];6(Supl 2):1-3. Disponível em: http://periodicos.unesc.net/Inovasaude/article/view/4216

20. Silva KM, Santos SMA. A consulta de enfermagem na estratégia de saúde da família: realidade de um distrito sanitário. Rev Enferm UFSM [Internet]. 2016 [cited 2019 Sept 10];6(2):248-58. Available from: https://periodicos.ufsm.br/reufsm/article/view/18079

21. Salci MA, Meirelles BHS, Silva DMGV. Primary care for diabetes mellitus patients from the perspective of the care model for chronic conditions. Rev Latinoam Enferm. 2017;25:e2882. doi: 10.1590/1518-8345.1474.2882

22. Imazu MFM, Faria BN, Arruda GO, Sales CA, Marcon SS. Efetividade das intervenções individual e em grupo junto a pessoas com diabetes tipo 2. Rev Latinoam Enferm. 2015;3(2):2007. doi: 10.1590/0104-1169.0247.2543

23. Bornstein VJ, Alencar A, Leandro BBS, Matielo E, Nespoli G, Goldschmidt IL, et al, organizadores. Curso de aperfeiçoamento em educação popular em saúde: textos de apoio [Internet]. Rio de Janeiro: EPSJV; 2016 [acesso em 2019 set 10]. Disponível em: http://www.ed popsus.epsjv.fiocruz.br/sites/default/files/conteudo/midia/arquivos/livroweb.pdf

24. Vasconcelos EM. Redefinindo as práticas de saúde a partir da educação popular nos serviços de saúde. In: Vasconcelos EM, Prado EV, organizadores. A saúde nas palavras e nos gestos: reflexões da rede de educação popular e saúde. $2^{\mathbf{a}}$ ed. São Paulo: Hucitec; 2017.

25. Lima LO, Pekelman R. O diálogo como estratégia formativa: perspectivas a partir da educação popular em saúde. Rev Educ Popul. 2020;N Esp:290-7. Disponível em: http://www.seer.ufu.br/index.php/reveducpop/article/view/56013/29438

26. Silva KS, Santos AM, Carvalho JA, Kochergin CN, Almeida PF. Percepção de gestores e 
enfermeiros sobre a organização do fluxo assistencial na rede de serviços de saúde. RECIIS. 2017;11(2):1-12. doi: 10.29397/reciis.v11i2.1226

Editora Científica: Tânia Solange Bosi de Souza Magnago

Editora Associada: Maria Denise Schimith

\section{Autor correspondente}

Valéria Silvana Faganello Madureira

E-mail: valeriamadureira2005@hotmail.com

Enderço: Rua Minas Gerais, 533E ap. 501, Bairro Presidente Médici, Chapecó, Santa Catarina, Brasil

CEP: 89.801-200

\section{Contribuições de Autoria}

\section{1 - Claudia Moresco Peliser Beal}

Elaboração do projeto de pesquisa; coleta, análise e discussão dos dados; autora do TCC que deu origem ao artigo.

Participou da redação do artigo.

\section{2 - Valéria Silvana Faganello Madureira}

Elaboração do projeto de pesquisa; acompanhamento da coleta, análise e discussão dos dados; orientadora do TCC que originou o artigo. Concepção, planejamento, redação e revisão do artigo.

\section{3 - Jeane Barros de Souza.}

Participou do planejamento, redação e revisão do artigo.

\section{4 - Liane Colliselli}

Participou da concepção, planejamento e redação do artigo.

\section{5 - Larissa Hermes Thomas Tombini}

Participou da redação do artigo.

\section{6 - Daniela Savi Geremia}

Participou da redação do artigo.

\section{Como citar este artigo}

Beal, Claudia Moresco Peliser; Madureira, Valéria Silvana Faganello; Souza, Jeane Barros de; Colliselli, Liane; Tombini, Larissa Hermes Thomas; Geremia, Daniela Savi. Cuidado de indivíduos com diabetes mellitus: a consulta de enfermagem na perspectiva de enfermeiras. Rev. Enferm. UFSM. 2020 [Acesso em: Anos Mês Dia]; vol.10 e92: 124. DOI: https://doi.org/10.5902/2179769242737 